\title{
Spatial fungal community diversity profiles in pit mud samples from fermentation cellars used to manufacture Chinese Strong-flavour liquor
}

\section{Shunchang $\mathrm{Pu}$}

Department of biology and food engineering, Bozhou University

\section{Cuie Shi}

School of Life Science, Huainan Normal University

\section{Yu Zhang}

Department of biology and food engineering, Bozhou University

Ning Lu

Department of biology and food engineering, Bozhou University

Shoubao Yan ( $\nabla 1176144756 @ q q . c o m$ )

School of Life Science, Huainan Normal University

\section{Research Article}

Keywords: fungal community, pit mud, volatile flavor compounds, fermentation

Posted Date: October 11th, 2021

DOI: https://doi.org/10.21203/rs.3.rs-855937/v1

License: (c) (1) This work is licensed under a Creative Commons Attribution 4.0 International License. Read Full License 


\section{Abstract \\ Background}

Few studies to date have sought to characterize the spatial profiles of pit mud microbial communities in fermentation cellars from Chinese strong-flavour liquor distilleries. This study was thus designed to evaluate these eukaryotic communities in pit mud samples via a multidimensional DGGE approach and by assessing associated sample physicochemical properties.

\section{Results}

Penicillium fuscoglaucum, Penicillium glandicola, Aotearoamyces nothofagi, Malassezia restricta, Penicillium lanosocoeruleum, Penicillium crustosum, and Aspergillus tonophilus were detected only in pit mud from the upper cellar wall, while Alternaria alstroemeriae, Trichosporon insectorum, Fusarium equiseti, Calonectria pseudoreteaudii, Penicillium clavigerum, Penicillium compactum, Ascochyta phacae, Metarhizium frigidum, Alternaria burnsii, Fusarium nurragi, and Didymella keratinophila were present only in the middle cellar wall layer. Alternaria zantedeschiae and llyonectria cyclaminicola were only present in pit mud samples from the lower cellar wall, while Leptobacillium leptobactrum, Calonectria queenslandica, Aspergillus appendiculatus, and Candida pseudolambica were only detected in pit mud from the cellar bottom. Moisture, $\mathrm{pH}, \mathrm{PO}_{4}{ }^{3-}$, acetic acid, humus, $\mathrm{K}^{+}, \mathrm{Mg}^{2+}, \mathrm{Ca}^{2+}$, acetic acid, butyric acid, and caproic acid levels in these different pit mud positions exhibited a rising incremental pattern from the upper wall layer to the bottom layer, whereas lactic acid levels were significantly lower in the bottom pit mud layer relative to other layers.

\section{Conclusions}

A clear relationship between fungal community structure and physicochemical variables in different spatial pit mud samples, especially moisture, $\mathrm{pH}$, and $\mathrm{NH}_{4}{ }^{+}-\mathrm{N}$ were identified as the three most significant factors associated with fungal community through a redundancy analysis.

\section{Background}

Chinese strong-flavour liquor is a traditional fermented beverage that accounts for roughly $70 \%$ of total liquor consumption in China [1]. Owing to its unique flavour and brewing approach, strong-flavour liquor holds a special status in Chinese culture and history. This liquor is distilled in large rectangular pit cellars ( $3600 \times 2300 \mathrm{~mm}$ at the top; $2800 \times 1540 \mathrm{~mm}$ at the bottom; $2400 \mathrm{~mm}$ deep) that serve as fermentation vessels (Fig. 1). The walls of these pits are covered with a specific type of fermented clay known as pit mud that contains large quantities of functional microorganisms including Clostridium spp., Bacillus spp., and Methanobacterium spp., all of which serve as key mediators of the fermentation process and sources of the aromatic compounds characteristic of Chinese strong-flavour liquor [2]. Indeed, the 
microbes within pit mud are generally accepted to play an essential role in the process of Chinese strongflavour liquor fermentation [3]. Given their importance, many studies have analyzed these microbial communities in an effort to better understand the mechanisms whereby these organisms contribute to the liquor production process [4].

Studies of pit mud conducted to date have primarily focused on prokaryotic flora [2]. For example, Liu et al. (2015) used a DGGE approach to explore Clostridium cluster I community diversity in samples of pit mud from cellars of different ages $(1,50,100$, and 400 years), revealing $C$. ragsdalei, $C$. ljungdahlii, $C$. autoethanogenum, and C. kluyveri to be the dominant species therein [3]. Liang et al. (2020) also employed a combination of PCR-DGGE and qPCR approaches to detect higher levels of Clostridium IV species in aged pit mud relative to aging pit mud, which they speculated may be associated with the fact that aged pit mud has a strong aroma whereas aging pit mud does not [5]. Ding et al. (2014)also conducted a nested PCR-DGGE-based study of eubacterial community structures in Chinese strongflavour liquor pit mud and found that community diversity was greater in the bottom of the cellar relative to in the cellar walls [6].

Few studies to date have sought to characterize the spatial profiles of pit mud microbial communities in fermentation cellars from Chinese strong-flavour liquor distilleries. This study was thus designed to evaluate these eukaryotic communities in pit mud samples via a multidimensional DGGE approach and by assessing associated sample physicochemical properties. In so doing, we aim to improve pit mud quality and consistency, and to facilitate the generation of artificial pit mud. by exploring pit mud microbial and physicochemical properties. This study is the first to our knowledge to have explored these multidimensional distributions of fungal communities and physicochemical properties in different spatial positions of pit mud by using PCR-DGGE methods.

\section{Results}

\section{DGGE profiling of fungal communities}

We began by characterizing the DGGE fingerprint profiles for pit mud fungal communities (Fig. 2). There were clear differences in the communities present within pit mud samples from the upper wall, middle wall, lower wall, and bottom cellar layers (Table 1). The Shannon-Wiener index value for the fungal community from the middle wall layer was greater than the corresponding values for the other analyzed pit mud samples, suggesting that maximal fungal diversity was present within this middle wall layer. The evenness index (E) values for these different fungal communities were between 0.961 and 0.996 , with these values being higher for samples from the middle wall and cellar bottom relative to other samples. Middle wall pit mud samples also exhibited the highest species richness index value, followed by samples from the bottom of the cellar, with no significant differences in these values when comparing samples from the upper or lower cellar wall. 
Table 1

Indices of fungal diversity in the samples collected from different spatial positions of cellar according to quantified bands from Fig. 2.

\begin{tabular}{|llll|}
\hline Lane $^{\text {a }}$ & Shannon-Wiener & Evenness & Richness \\
\hline $\mathrm{U}$ & 3.17 & 0.989 & 25 \\
\hline $\mathrm{M}$ & 3.69 & 0.996 & 41 \\
\hline $\mathrm{D}$ & 3.15 & 0.993 & 24 \\
\hline B & 3.45 & 0.996 & 32 \\
\hline $\begin{array}{l}\text { a Lane N represent samples collected from NFTSW; Lanes U, M, D, and B respectively represent pit } \\
\text { mud samples collected from up wall layer of cellar, middle wall layer of cellar, down wall layer of } \\
\text { cellar, and bottom layer of cellar, and were sampled from the same fermentation cellar. }\end{array}$ \\
\hline
\end{tabular}


Table 2

BLAST Identified gene sequences of ITS - derived bands excised from a DGGE gel

\begin{tabular}{|c|c|c|}
\hline Band no. ${ }^{a}$ & Closest relative (NCBI accession no.) & Identity (\%) \\
\hline 1 & Penicillium fuscoglaucum (NR_163669.1) & 97.25 \\
\hline 2 & Penicillium glandicola (MH860946.1) & 97.40 \\
\hline 3 & Alternaria alstroemeriae (MH863036.1) & 99.61 \\
\hline 4 & Trichosporon insectorum (MW433667.1) & 98.54 \\
\hline 5 & Simplicillium chinense (MK102638.1) & 100.00 \\
\hline 6 & Leptobacillium leptobactrum (MG786580.1 ) & 97.04 \\
\hline 7 & Penicillifer martinii (KJ869167.1) & 96.19 \\
\hline 8 & Calonectria queenslandica (NR_121455.1) & 97.59 \\
\hline 9 & Alternaria doliconidium (MT672468.1) & 100.00 \\
\hline 10 & Ramgea ozimecii (KY368752.1) & 96.94 \\
\hline 11 & Alternaria destruens (DQ323680.1) & 100.00 \\
\hline 12 & Aotearoamyces nothofagi (MG807392.1) & 96.79 \\
\hline 13 & Alternaria helianthiinficiens (MF414166.1) & 96.42 \\
\hline 14 & Fusarium equiseti (KX463025.1) & 99.59 \\
\hline 15 & Fusarium circinatum (NR_120263.1) & 96.14 \\
\hline 16 & Epicoccum phragmospora (MW237699.1) & 96.92 \\
\hline 17 & Alternaria zantedeschiae (MH864493.1) & 96.66 \\
\hline 18 & Bipolaris axonopicola (KX452443.1) & 97.56 \\
\hline 19 & Metarhizium robertsii (NR_132011.1) & 96.21 \\
\hline 20 & Calonectria pseudoreteaudii (NR_137040.1) & 96.64 \\
\hline 21 & Alternaria betae-kenyensis (NR_136118.1) & 98.19 \\
\hline 22 & Cladosporium chasmanthicola (NR_152307.1) & 100.00 \\
\hline 23 & Seltsamia ulmi (NR_156634.1) & 96.38 \\
\hline 24 & Trichosporon inkin (NR_073243.1) & 98.51 \\
\hline
\end{tabular}

a Numbers are those of bands shown in Fig. 2 .

b Most homologous BLAST-derived match. 


\begin{tabular}{|c|c|c|}
\hline Band no. ${ }^{a}$ & Closest relative (NCBI accession no.) & Identity $(\%)^{\mathrm{b}}$ \\
\hline 25 & Trichosporon coremiiforme(NR_073249.1) & 98.03 \\
\hline 26 & Penicillium clavigerum (NR_121317.1) & 96.52 \\
\hline 27 & Penicillium roqueforti (NR_103621.1) & 100.00 \\
\hline 28 & Malassezia restricta (NR_103585.1) & 98.88 \\
\hline 29 & Penicillium caseifulvum (NR_163685.1) & 96.34 \\
\hline 30 & Penicillium compactum (NR_144844.1) & 96.33 \\
\hline 31 & Penicillium lanosocoeruleum (NR_163541.1) & 96.78 \\
\hline 32 & Penicillium crustosum (NR_077153.1) & 96.69 \\
\hline 33 & Aspergillus intermedius (NR_137448.1) & 99.01 \\
\hline 34 & Pichia kudriavzevii (NR_131315.1) & 98.15 \\
\hline 35 & Alternaria arborescens (NR_135927.1) & 100.00 \\
\hline 36 & Ascochyta phacae (KT389475.1) & 96.62 \\
\hline 37 & Aspergillus tonophilus (NR_137450.1) & 97.54 \\
\hline 38 & Penicillium argentinense (NR_121523.1) & 96.89 \\
\hline 39 & Metarhizium frigidum (NR_132012.1) & 96.02 \\
\hline 40 & Alternaria burnsii (NR_136119.1) & 99.10 \\
\hline 41 & Alternaria radicina ATCC (NR_165503.1) & 97.18 \\
\hline 42 & Aspergillus appendiculatus (NR_135433.1) & 96.91 \\
\hline 43 & Thermomyces lanuginosus (NR_121309.1) & 99.69 \\
\hline 44 & Penicillium robsamsonii (NR_144866.1) & 96.58 \\
\hline 45 & Antarctomyces psychrotrophicus (NR_164292.1) & 97.47 \\
\hline 46 & Fusarium nurragi (NR_159860.1) & 97.75 \\
\hline 47 & Didymella keratinophila (NR_158275.1) & 97.72 \\
\hline 48 & Aspergillus heterocaryoticus (NR_163674.1) & 100.00 \\
\hline 49 & Penicillium citrinum (NR_121224.1) & 99.50 \\
\hline 50 & Ilyonectria cyclaminicola (NR_121495.1) & 97.27 \\
\hline \multicolumn{3}{|c|}{ a Numbers are those of bands shown in Fig. 2 . } \\
\hline b Most hor & logous BLAST-derived match. & \\
\hline
\end{tabular}




\begin{tabular}{|lll|}
\hline Band no. $^{\text {a }}$ & Closest relative (NCBI accession no.) & Identity (\%) \\
\hline 51 & Candida pseudolambica (NR_153281.1) & 97.53 \\
\hline a Numbers are those of bands shown in Fig. 2. & \\
\hline b Most homologous BLAST-derived match. \\
\hline
\end{tabular}

UPGMA dendrograms were constructed for DGGE profiles based upon Dice coefficient values in order to describe community similarity between pit mud samples from different positions within the fermentation cellar (Fig. 3). Cluster analyses of these fungal profiles revealed that pit mud samples from the upper wall layer formed a group, while the primary microbial populations present in samples from the lower wall layer were similar to those in pit mud samples from the cellar bottom (Fig. 3).

To more fully understand the dominant fungi within pit mud samples, DGGE profile bands were carefully excised, purified, and sequenced (Table 2, Fig. 2). In total, 51 bands were sequenced, with the resultant sequences having a similarity of $96 \%$ to those in the GenBank database. These ITS sequences were associated with 25 fungal genera: Penicillium, Alternaria, Trichosporon, Simplicillium, Leptobacillium, Penicillifer, Calonectria, Ramgea, Aotearoamyces, Fusarium, Epicoccum, Bipolaris, Metarhizium, Cladosporium, Seltsamia, Malassezia, Aspergillus, Pichia, Ascochyta, Thermomyces, Antarctomyces, Fusarium, Didymella, Ilyonectria, and Candida. The two dominant genera in these samples were Aspergillus and Alternaria species, which accounted for $21.57 \%$ and $15.69 \%$ of the identified fungi, respectively.

As shown in Fig. 2 and Fig. 4, Alternaria doliconidium (band 9), Ramgea ozimecii (band 10), Alternaria destruens (band 11), Alternaria betae-kenyensis (band 21), Cladosporium chasmanthicola (band 22), Seltsamia ulmi (band 23), and Penicillium argentinense (band 38) were present in all pit mud samples, with Alternaria destruens (band 11) and Alternaria doliconidium (band 9) being present at notably high levels, suggesting that they may be dominant members of the pit mud flora and that they may be key mediators of liquor fermentation, although additional research will be needed to test this possibility. In contrast, Penicillium fuscoglaucum (band 1), Penicillium glandicola (band 2), Aotearoamyces nothofagi (band 12), Malassezia restricta (band 28), Penicillium lanosocoeruleum (band 31), Penicillium crustosum (band 32), and Aspergillus tonophilus (band 35) were only detected in the pit mud layer form the upper cellar wall, whereas Alternaria alstroemeriae (band 3), Trichosporon insectorum (band 4), Fusarium equiseti (band 14), Calonectria pseudoreteaudii (band 20), Penicillium clavigerum (band 26), Penicillium compactum (band 30), Ascochyta phacae (band 36), Metarhizium frigidum (band 39), Alternaria burnsii (band 40), Fusarium nurragi (band 46), and Didymella keratinophila (band 47) were only present in the middle cellar wall. Similarly, Alternaria zantedeschiae (band 17), and Ilyonectria cyclaminicola (band 50) were only detected in pit mud samples from the lower cellar wall, while Leptobacillium leptobactrum (band 6), Calonectria queenslandica (band 8), Aspergillus appendiculatus (band 42), and Candida pseudolambica (band 51) were only present in samples from the bottom pit mud layer. Antarctomyces psychrotrophicus (band 45), and Aspergillus heterocaryoticus (band 48) were present at high levels in the 
middle wall, lower wall, and bottom pit mud layers. Trichosporon inkin (band 24) was present in all three wall layers from the same cellar, while Simplicillium chinense (band 5), Trichosporon coremiiforme (band 25), and Aspergillus intermedius (band 33) were only evident in the upper and middle wall layers.

Penicillifer martinii (band 7), Fusarium circinatum (band 15), Epicoccum phragmospora (band 16), and Bipolaris axonopicola (band 18) were present in the middle wall and cellar bottom pit mud layers. Penicillium caseifulvum (band 29) was only found in the upper wall, middle wall, and cellar bottom pit mud layers, whereas Metarhizium robertsii (band 19), Penicillium roqueforti (band 27), and Pichia kudriavzevii (band 34) were present in the upper wall layer and the bottom layer. Alternaria radicina (band 41) and Alternaria radicina (band 49) were only found in the middle and lower wall layers, and Alternaria helianthiinficiens (band 13) was detected in the lower wall and bottom layers.

\section{Physiochemical properties}

The physicochemical properties of pit mud samples from different cellar positions were next evaluated (Table 3). Levels of moisture, $\mathrm{pH}_{1} \mathrm{PO}_{4}{ }^{3-}$, acetic acid, Humus, $\mathrm{K}^{+}, \mathrm{Mg}^{2+}, \mathrm{Ca}^{2+}$, acetic acid, butyric acid, and caproic acid, changed incrementally with position from the upper wall layer to the deepest bottom pit mud layer, consistent with the gradient-like distribution of these physicochemical attributes in 20-year-old pit mud, as previously demonstrated by Meng et al. (2020) [7]. Levels of $\mathrm{NH}_{4}{ }^{+} \mathrm{N}$ were higher in the bottom pit mud layer relative to other layers, whereas these levels did not differ significantly between the middle and bottom wall pit mud layers, and were lowest in the upper wall layer pit mud samples. In contrast, lactic acid levels exhibited the opposite trend such that these levels were significantly lower in the bottom pit mud wall layer. 
Table 3

The physicochemical properties of pit mud samples from different spatial positions of cellar

\begin{tabular}{|c|c|c|c|c|}
\hline Parameter & $\mathbf{U}$ & M & D & B \\
\hline Moisture (\%) & $32.54 \pm 2.65$ & $35.11 \pm 1.51$ & $37.68 \pm 2.57$ & $39.35 \pm 2.15$ \\
\hline $\mathrm{pH}$ & $5.23 \pm 0.25$ & $5.45 \pm 0.16$ & $7.56 \pm 0.46$ & $9.23 \pm 0.56$ \\
\hline $\mathrm{NH}^{4+}-\mathrm{N}(\mathrm{g} / \mathrm{kg})$ & $2.06 \pm 0.21$ & $3.98 \pm 0.29$ & $4.08 \pm 0.35$ & $5.28 \pm 0.37$ \\
\hline $\mathrm{PO}^{3-}{ }^{3-}(\mathrm{mg} / \mathrm{kg})$ & $201.35 \pm 15.32$ & $256.35 \pm 20.31$ & $335.26 \pm 28.35$ & $387.65 \pm 30.21$ \\
\hline Humus (\%) & $5.35 \pm 0.34$ & $9.024 \pm 0.87$ & $10.31 \pm 0.89$ & $15.56 \pm 1.32$ \\
\hline $\mathrm{K}^{+}(\mathrm{mg} / \mathrm{kg})$ & $525.35 \pm 46.72$ & $678.54 \pm 52.08$ & $834.21 \pm 54.32$ & $1125.35 \pm 67.25$ \\
\hline $\mathrm{Mg}^{2+}(\mathrm{mg} / \mathrm{kg})$ & $134.65 \pm 69.17$ & $181.45 \pm 56.23$ & $201.32 \pm 68.45$ & $245.32 \pm 78.65$ \\
\hline $\mathrm{Ca}^{2+}(\mathrm{mg} / \mathrm{kg})$ & $368.32 \pm 13.54$ & $438.57 \pm 25.21$ & $517.36 \pm 23.56$ & $708.19 \pm 47.43$ \\
\hline $\begin{array}{l}\text { Acetic acid } \\
(\mathrm{mg} / \mathrm{kg})\end{array}$ & $556.54 \pm 46.28$ & $677.35 \pm 58.32$ & $856.37 \pm 75.64$ & $1235.94 \pm 98.56$ \\
\hline $\begin{array}{l}\text { Butyric acid } \\
(\mathrm{mg} / \mathrm{kg})\end{array}$ & $397.86 \pm 32.82$ & $623.74 \pm 58.08$ & $926.48 \pm 86.37$ & $1021.87 \pm 90.89$ \\
\hline $\begin{array}{l}\text { Caproic acid } \\
(\mathrm{mg} / \mathrm{kg})\end{array}$ & $2356.54 \pm 120.37$ & $3570.35 \pm 234.52$ & $5256.37 \pm 136.85$ & $7563.25 \pm 163.21$ \\
\hline $\begin{array}{l}\text { Lactic acid } \\
(\mathrm{mg} / \mathrm{kg})\end{array}$ & $25348.89 \pm 875.89$ & $18692.32 \pm 785.65$ & $13897.87 \pm 567.31$ & $11783.41 \pm 710.65$ \\
\hline
\end{tabular}

\section{Relationships between fungal communities and physicochemical variables}

A redundancy analysis (RDA) was next conducted to better clarify potential relationships between the 51 detected fungal genera and the 12 analyzed environmental factors (Fig. 5). The first two component axes explained $77.6 \%$ of the variation in fungal composition, with species-specific environmental correlations for both axes of $48.1 \%$ and $78.6 \%$, respectively, indicating that fungal community structure was moderately correlated with these physicochemical variables. An interactive forward selection procedure was used to evaluate these 12 environmental variables, revealing that moisture, $\mathrm{pH}$, and $\mathrm{NH}_{4}{ }^{+}-\mathrm{N}$ contributed significantly to community composition $(39.5 \%, 13.8 \%$, and $13.8 \%$, respectively; $P<0.01)$, whereas the other 8 variables exhibited no significant correlations. 
As shown in Fig. 5, AZA (Alternaria zantedeschiae), ICY (Ilyonectria cyclaminicola), CPE (Calonectria pseudoreteaudii), LLE (Leptobacillium leptobactrum), CQU (Calonectria queenslandica), AAP (Aspergillus appendiculatus), AHT (Aspergillus heterocaryoticus), PAR (Penicillium argentinense), APS (Antarctomyces psychrotrophicus), and ROZ (Ramgea ozimecii) were strongly positively correlated with moisture, $\mathrm{pH}, \mathrm{NH}_{4}{ }^{+}-\mathrm{N}, \mathrm{PO}_{4}{ }^{3-}$, Humus, $\mathrm{K}^{+}, \mathrm{Mg}^{2+}, \mathrm{Ca}^{2+}$, acetic acid, butyric acid, and caproic acid. In addition, MRO (Metarhizium robertsii), ADE (Alternaria destruens), BAX (Bipolaris axonopicola), TLA (Thermomyces lanuginosus), and $\mathrm{CCH}$ (Cladosporium chasmanthicola) were moderately positively correlated with these variables, while correlations were weaker for EPH (Epicoccum phragmospora), PMA (Penicillifer martinii), AHE (Alternaria helianthiinficiens), and $\mathrm{FCl}$ (Fusarium circinatum). As shown in the upper portion of Figure 5, TIN (Trichosporon inkin), and ADO (Alternaria doliconidium) were closely associated with lactic acid, while ANO (Aotearoamyces nothofagi), PFU (Penicillium fuscoglaucum), PLA (Penicillium lanosocoeruleum), MRE (Malassezia restricta), PGL (Penicillium glandicola), PCR (Penicillium crustosum), AAR (Alternaria arborescens), PRO (Penicillium robsamsonii), PRQ (Penicillium roqueforti), PKU (Pichia kudriavzevii), TCO (Trichosporon coremiiforme), SUL (Seltsamia ulmi), SCH (Simplicillium chinense), AIN (Aspergillus intermedius), PCA (Penicillium caseifulvum), APH (Ascochyta phacae), ATO (Aspergillus tonophilus), PCO (Penicillium compactum), DKE (Didymella keratinophila), PCI (Penicillium citrinum), MFR (Metarhizium frigidum), CPS (Candida pseudolambica), ABU (Alternaria burnsii), TIN (Trichosporon insectorum), FNU (Fusarium nurragi), FEQ (Fusarium equiseti), and AAL (Alternaria alstroemeriae) were only weakly correlated with this variable.

\section{Discussion}

Chinese strong-flavour liquor is prepared through the fermentation of a mixture of sorghum, rice, and wheat known as Zaopei in a rectangular cellar composed of pit mud. This pit mud is an ideal habitat for microbes that are integral to the distillation process, serving as key determinants of the flavour of the resultant liquor. The quality of pit mud is thus an important regulator of the quality and taste of the liquor produced.

Pit mud tends to age with increasing cellar usage, and the microbial communities present within this mud vary based upon their spatial location within the walls or bottom of the cellar. A range of sensory descriptions and physicochemical indices have been used to describe pit mud from different locations within these fermentation cellars. For example, pit mud from the bottom of these cellars is often described as smooth, fine, soft, moist, and sooty with an aroma of esters, ammonia, and hydrogen sulfide. In contrast, pit mud from the top of these cellars is rough, hard, dry, and light grey with white lumps or aciform crystals and no aroma. While pit mud from the bottom layer can support the production of good-quality liquor, that from the upper layer cannot. As such, studying the microbial communities present within pit mud is essential in order to understand the molecular mechanisms governing the flavor and aroma of Chinese strong-flavour liquor in an effort to improve the quality of this popular and culturally important beverage. 
In prior studies, researchers have utilized both culture-dependent and -independent strategies to determine that bacteria, fungal, archaea, and actinomycetes species are present within pit mud samples, with bacteria and archaea being dominant in this environment [5]. At the family level, common pit mudresident bacteria include haloplasmatacea, Bacillaceae, planococcaceae, synergistaceae, staphylococcaceae, Thermoanaerobacter, and clostridiaceae species. Archaea present within pit mud are largely consistent across regions, and primarily include methanobacteria (Methanobacteriaceae), Methanococcus (Methanococcus), and thermoplasmataceae (thermoplasmata) species [5]. Microbes in the Clostridia class are thought to be primary producers of short- and medium-chain fatty acids including butanoic acid and hexanoic acid, which are directly relevant to the liquor production process [4]. Liu et al. (2018) isolated the Lysi-nibacillussphaerieus, Brevibacillusbrevis, and Paenibacilluslarvae subsup.pulvifaciens strains from pit mud and found that these microbes were important mediators of fermentation and producers of aromatic compounds in the context of Chinese strong-flavour liquor distillation [8]. Wang et al. (2019) explored bacterial community structures in samples of pit mud from a 16-year-old Chinese strong-flavour liquor cellar, and found that Clostridium, Aminobacterium, Petrimonas, Syntrophmonas, and Sedimentibacter species were the dominant microbes therein [9]. Ding et al. (2014) employed a PCR-DGGE approach to characterize the eubacterial pit mud communities associated with Luzhou-flavour liquor and consistently detected higher levels of eubacterial diversity in samples from the bottom of the cellar relative to samples from the cellar walls [6]. Most of these past studies have specifically focused on prokaryotic species, and there have been few comparable analyses of the fungal communities found within pit mud.

Herein, we explored the structures of fungal communities in multidimensional pit mud environments via a DGGE approach, revealing clear discrimination between the communities present in different locations within the fermentation cellar. Penicillium fuscoglaucum, Penicillium glandicola, Aotearoamyces nothofagi, Malassezia restricta, Penicillium lanosocoeruleum, Penicillium crustosum, and Aspergillus tonophilus were only present in the upper cellar wall pit mud layer, whereas Alternaria alstroemeriae, Trichosporon insectorum, Fusarium equiseti, Calonectria pseudoreteaudii, Penicillium clavigerum, Penicillium compactum, Ascochyta phacae, Metarhizium frigidum, Alternaria burnsii, Fusarium nurragi, and Didymella keratinophila were presented only detected in the middle wall layer. Similarly, Alternaria zantedeschiae and Ilyonectria cyclaminicola were only identified in the lower cellar wall pit mud layer, while Leptobacillium leptobactrum, Calonectria queenslandica, Aspergillus appendiculatus, and Candida pseudolambica were only detected in the pit mud found on the bottom of the fermentation cellar. These differences may explain why the quality of strong-flavour liquor varies with cellar position. We found that fungal abundance in the upper and middle layers was significantly higher than that in the lower wall and bottom layers, potentially due to the lower oxygen levels in these latter two environments, as such oxygen deficiency may have compromised fungal survival [10]. This, in turn, may explain the higher saccharification efficiency that is typically detected in the upper and middle Zaopei layers in the context of liquor fermentation. 
With respect to pit mud physicochemical properties, we found that moisture, $\mathrm{pH}, \mathrm{PO}_{4}{ }^{3-}$, acetic acid, Humus, $\mathrm{K}^{+}, \mathrm{Mg}^{2+}, \mathrm{Ca}^{2+}$, acetic acid, butyric acid, and caproic acid levels rose with sample position from the upper wall to the bottom of the fermentation cellar, suggesting that organic compounds were gradually degraded with position. The maximal moisture levels in the bottom pit mud layer may be associated with the high levels of Huangshui present in this setting. The higher $\mathrm{pH}$ levels lower in the cellar may be linked to the degradation of various acids such as lactic acid [9], and to the synthesis of ammonium nitrogen, consistent with the observed trends in $\mathrm{NH}_{4}{ }^{+} \mathrm{N}$ levels. The lower acetic acid levels with the upper wall pit mud layer are consistent with less robust prokaryotic metabolism in this location, given that acetic acid is a metabolic end product produced by many bacterial species [4]. The rising lactic acid levels detected from the bottom of the pit to the upper pit may correspond to the different Lactobacillus activity levels in these positions.

We then conducted an RDA analysis to explore relationships between the 51 detected fungal genera and the 12 measured environmental variables (Fig. 4). This revealed that moisture, $\mathrm{pH}$, and $\mathrm{NH}_{4}{ }^{+}-\mathrm{N}$ levels were the most significant environmental factors, accounting for $67.1 \%$ of microbial community variability, indicating that these physicochemical factors are closely linked to pit mud microbe growth. Fungal community structure was moderately correlated with these physicochemical variables. For example, Alternaria zantedeschiae, Ilyonectria cyclaminicola, Calonectria pseudoreteaudii, Leptobacillium leptobactrum, Calonectria queenslandica, Aspergillus appendiculatus, Aspergillus heterocaryoticus, Penicillium argentinense, Antarctomyces psychrotrophicus, and Ramgea ozimecii levels were strongly positively correlated with moisture, $\mathrm{pH}_{1} \mathrm{NH}_{4}{ }^{+}-\mathrm{N}, \mathrm{PO}_{4}{ }^{3-}$, Humus, $\mathrm{K}^{+}, \mathrm{Mg}^{2+}, \mathrm{Ca}^{2+}$, acetic acid, butyric acid, and caproic acid levels, whereas for Metarhizium robertsii, Alternaria destruens, Bipolaris axonopicola, Thermomyces lanuginosus, and Cladosporium chasmanthicola levels these correlations were more moderate.

Many prior studies have sought to understand the relationship between pit mud physicochemical properties and the microbial communities therein. Meng et al. (2020), for example, found that these properties were significantly influenced by depth within the fermentation cellar [7]. Zhang et al. (2020) found that acid and amino nitrogen concentrations were higher in the bottom pit mud layers relative to other positions, suggesting that these compounds may influence the overall diversity of the microbial communities found within this bottom layer [4]. We similarly detected a clear relationship between fungal community structure and physicochemical variables in pit mud samples. However, further research will be essential to develop the efficient cultivation strategies necessary to delineate the independent contributions of different fungi to the production of Chinese strong-flavour liquor production.

\section{Conclusions}

This study explored the multidimensional distributions of fungal communities and physicochemical properties in different spatial positions of pit mud by using PCR-DGGE methods. There were clear differences in the fungal communities present within pit mud samples from the upper wall, middle wall, 
lower wall, and bottom cellar layers. RDA analysis demonstrated that a clear relationship between fungal community structure and physicochemical variables in different spatial pit mud samples, especially moisture, $\mathrm{pH}$, and $\mathrm{NH}_{4}{ }^{+}-\mathrm{N}$ were identified as the three most significant factors associated with fungal community through a redundancy analysis. This study provide theoretical basis to design effective strategies to manipulate microbial consortia for better improving pit mud quality in Chinese strongflavour liquor production.

\section{Materials And Methods Sample collection}

Samples of pit mud were obtained from 20-year-old pits from a well-known liquor manufacturer (Anhui Yingjia Distillery Group Co., Ltd.) located in Luan city, Anhui province, China. Sampling sites are detailed in Fig. 1. Sampling was conducted as per a stratified random approach [11]. Pit mud wall samples were collected from the center of each wall, with approximately $50 \mathrm{~g}$ of mud being collected per position and mixed to yield a composite sample. Samples of pit mud from the cellar bottom were collected from all corners and the center of each pit, and were mixed together. All samples were collected at a depth of $\sim 5$ $\mathrm{cm}$. Samples of mixed pit mud from these different sampling sites were separated into small $\sim 100 \mathrm{~g}$ samples and stored at $-20^{\circ} \mathrm{C}$ prior to analysis.

\section{Physiochemical property analyses}

Pit mud moisture levels were established by drying samples for $3 \mathrm{~h}$ at $115^{\circ} \mathrm{C}$. Pit mud $\mathrm{pH}$ values were established with a Mettler Toledo pH meter after diluting sample 1:4 (w/v) with $\mathrm{ddd}_{2} \mathrm{O}$. for $3 \mathrm{~h}$. Pit mud ammonium $\left(\mathrm{NH}_{4}{ }^{+}-\mathrm{N}\right)$ levels were established via extraction in $10 \%(\mathrm{w} / \mathrm{v}) \mathrm{NaCl}$ at 1:10 (w/v) ratio, after which concentrations were measured using a UV spectrophotometer. Acetic acid, butyric acid, and caproic acid were extracted using 15\% methanol and quantified via gas chromatography (Agilent 7890, US) as described previously [12]. Lactic acid (LA) levels were quantified via utra-high-performance liquid chromatography (UPLC, Acquity I-class, Waters, US) as previously reported [1]. Levels of $\mathrm{K}^{+}, \mathrm{PO}_{4}{ }^{3-}$, soluble $\mathrm{Mg}^{2+}$, and soluble $\mathrm{Ca}^{2+}$ in air-dried pit mud were measured via extracting samples with $\mathrm{ddH}_{2} \mathrm{O}$ at a 1:10 $(\mathrm{w} / \mathrm{v})$ ratio, after which concentrations were measured as reported previously by Zhang et al. (2020) using an ion chromatograph (ICS5000 ${ }^{+}$, ThermoFisher) equipped with a conductivity detector (ICS-5000+-DC) and a CS12 column (IonPac, ThermoFisher, $4 \mathrm{~mm} \times 250 \mathrm{~mm}$ ) [4]. The utilized injection volume was $25 \mu \mathrm{L}$, with methane sulfonic acid $(20 \mathrm{mM})$ as a carrier fluid at a $1 \mathrm{~mL} / \mathrm{min}$ flow rate, with a column temperature of $30^{\circ} \mathrm{C}$. Humus levels were determined as detailed previously by Shen (2007) [13].

\section{Fungal community assessment}

\section{DNA extraction}

A Fast DNA SPIN Kit for Soil (MP Biomedicals, OH, USA) was used based on provided directions to extract DNA from pit mud samples. Samples were analyzed in triplicate. Briefly, a Mini-Beadbeater (Biospec 
Products, OK, USA) was used to homogenize samples for $1 \mathrm{~min}$ at $5000 \mathrm{rpm}$, after which DNA was eluted in TE buffer (10 mM Tris-HCl, $1.0 \mathrm{mM}$ EDTA), diluted 20-fold with this same buffer, and analyzed.

\section{PCR amplification}

The ITS gene was first amplified with the universal ITS1f (5'-CTTGGTCATTTAGAGGAAGTAA-3') and ITS4 (5'-TCCTCCGCTTATTGATATGC-3') primers as detailed by Cobo-Díaz et al. (2019)[14], after which the nested PCR NS2 (5'-GCTGCGTTCTTCATCGATGC-3') and the GC-clamp 5'-

CGCCCGCCGCGCGCGGCGGGCGGGGCGGGGGCACGGGGGGCTTGGTCATTTAGAGGAAGTAA-3') primers as detailed previously [15]. Prior to DGGE analysis, samples were analyzed via 1\% agarose gel electrophoresis.

\section{DGGE analysis}

The BioRad DCode Universal Mutation Detection System (BioRad, CA, USA) was used for DGGE analyses with $8 \%(\mathrm{w} / \mathrm{v})$ polyacrylamide gels in $1 \times \mathrm{TAE}$. A $30-50 \%$ urea-formamide denaturing gradient (diluted from a $7 \mathrm{M}$ urea and $40 \%(\mathrm{w} / \mathrm{v})$ formamide stock) yielded optimal fungal sample separation. Gels were run for $17 \mathrm{~h}$ at $100 \mathrm{~V}$ at $60^{\circ} \mathrm{C}$, after which they were stained with $\mathrm{AgNO}_{3}$ as published previously [16]. The Quantity One software and a calibrated imaging densitometer GS-710 (Bio-Rad) were then used to image and analyze DGGE fingerprint profiles.

\section{DGGE band sequencing}

Representative DGGE were excised with a sterile scalpel, and were added to ultrapure water overnight at $4^{\circ} \mathrm{C}$ to facilitate sample elution. Samples from eluted bands were then again amplified with the GC-clamp primers detailed above, After amplification, samples were again assessed via DGGE gels to confirm purity. Bands were then re-amplified using the same primers without the GC clamp, and were purified using a universal PCR purification kit (Sangon, Shanghai, China). Cloning and sequencing were then performed by Sangon, and the resultant sequences were compared to ITS sequences in the GenBank (http://www.ncbi.nlm.nih.gov) databases to identify the closest phylogenetic relatives.

\section{Data analysis}

Cluster and community diversity analyses were performed with the Quantity One software, with individual DGGE lanes being converted into densitometric profiles. Fungal community Shannon-Wiener index of general diversity $(H)$, the Evenness $(E)$, and the richness $(S)$ values were then calculated based upon relative band intensity with the PAST software package (Palaeontology Statistics, http://folk.uio.no/ohammer/past/). The unweighted pair group method with arithmetic averages (UPGMA) was used for sample clustering.

\section{Declarations}

\section{Ethics approval and consent to participate}

Not applicable. 


\section{Consent for publication}

Not applicable.

\section{Availability of data and materials}

The datasets used and/or analysed during the current study are available from the corresponding author on reasonable request.

\section{Competing interests}

The authors of this manuscript declare they have no conflict of interest.

\section{Funding}

This research was funded by the Key Program in the Youth Elite Support Plan in Universities of Anhui Province (Nos. gxyqZD2016523); the academic funding for top talents in disciplines (Specialties) of Anhui Provincial Higher Education Institutes (Nos. gxbjZD2021087); the Key Research and Development Project of Anhui Province (Nos. 1804a07020122, and1704f0704067); the academic funding for top talents in disciplines (Specialties) of Anhui Provincial Higher Education Institutes (Nos. gxbjZD35).

\section{Authors' contributions}

SP, CS, and SY were mostly involved in laboratory works and data analysis and writing the first draft. YZ and NL had the main contribution in the study's conception and design, revising, and approving the first draft. All authors have read and agreed to the published version of the manuscript.

\section{Acknowledgements}

The authors would like to thank Anhui Yingjia Co., Ltd. for its support in this work.

\section{Authors' information}

${ }^{1}$ Department of biology and food engineering, Bozhou University, Bozhou, Anhui 236800, People's Republic of China. ${ }^{2}$ School of Life Science, Huainan Normal University, Huainan, Anhui 232001, People's Republic of China.

\section{References}

1. Yan, S.B., Wang, S.C, Wei, G.G., \& Zhang, K.G. Investigation on the main parameters during the fermentation of Chinese Luzhou-flavor liquor. Journal of the institute of brewing.2015, 121: 145154,doi: 10.1002/jib.193.

2. Gao, Z.Z., Wu, Z.Y., \& Zhang, W.X. Research progress on microbial community in pit mud of strongflavor Baijiu. China brewing.2020, 39(6), 1-7,doi: 10.11882/j.issn.0254-5071.2020.06.001. 
3. Liu, M.K., Zhao, K., Tang, Y. M., Ren, D.Q., Yao, W.C., Tian, X.H., Zhang, X.Y., Yi, B. \& Deng, B. Analysis of Clostridium Cluster I Community Diversity in Pit Mud Used in Manufacture of Chinese Luzhouflavor Liquor. Food science and biotechnology.2015, 24(3), 995-1000,doi: 10.1007/s10068-015-01277.

4. Zhang, H.M., Meng, Y.J., Wang, Y.L., Zhou, Q.W., Li, A.J., Liu, G.Y., Li, J.X., \& Xing X.H.Prokaryotic communities in multidimensional bottom-pit-mud from old and young pits used for the production of Chinese Strong-Flavor Baijiu. Food Chemistry.2020, 312, 126084,doi:

10.1016/j.foodchem.2019.126084.

5. Liang, H., Xu, C.F., Zhu, L., \& Tang, W.B. Research progress on regional characteristics and pit mud microorganisms of strong-flavor Baijiu. China brewing.2020, 39(11), 5-8,doi: 10.11882/j.issn.02545071.2020.11.002.

6. Ding, X.F., Wu, C.D., Zhang, L.Q., Zheng, J., \& Zhou, R.Q. Characterization of eubacterial and archaeal community diversity in the pit mud of Chinese Luzhou flavor liquor by nested PCR-DGGE. World Journal of Microbiology and Biotechnology.2014, 30(2), 605-612,doi: 10.1007/s11274-013-1472-4.

7. Meng, Y.J., Zhang, H.M., Wang, Y.L., Liang, J.H., Zhou, Q.W., Wang, Y.H., Liu, G.Y., He, H.K., \& Li, A.J. Eukaryotic Community Structure of Strong Flavor Baijiu Mud. Modern Food Science and Technology.2020, 36(5), 96-101, doi: 10.13982/j.mfst.1673-9078.2020.5.022.

8. Liu, Y.M., Wang, Y.L., Li, Y.B., Wang, W.P., Liu, M., Wu, S.K., Huang, Z.G., \& Li, H. The study of microorganisms in Pit Mud by Agilent GC and GC-MS. Environmental Chemistry.2018, 37(4): 902905,doi: CNKI:SUN:HJHX.0.2018-04-033.

9. Wang, C.Y., Song, J.Y., Lv, H.X., Zhang, Y.M., Fan, Y.P., Li, X.S., \& Guo, S.X. Analysis of bacterial community structure in strong-flavour Baijiu pit mud with different cellar ages from Songhe distillery based on high-throughput sequencing. China brewing.2019,38(8),163-166,doi: 10.11882/j.issn. 02545071.2019.09.031.

10. Zheng, J., Liang, R., Zhang, L.Q., Wu, C.D., Zhou, R.Q., \& Liao, X.P. Characterization of microbial communities in strong aromatic liquor fermentation pit muds of different ages assessed by combined DGGE and PLFA analyses. Food Research International.2013, 54, 660-666,doi: 10.1016/j.foodres.2013.07.05.

11. Carter, M. R., \& Gregorich, E. G. Soil sampling and methods of analysis (2nd ed.) Boca Raton: CRC Press, 2006,Taylor \& Francis Group.

12. Liu, M.K., Tang, Y. M., Guo, X. J., Zhao, K., Tian, X. H., Liu, Y., \& Zhang, X. P. Deep sequencing reveals high bacterial diversity and phylogenetic novelty in pit mud from Luzhou Laojiao cellars for Chinese strong-flavor Baijiu. Food Research Internationa.2017l, 102, 68-76, doi: 10.1016/j.foodres.2017.09.075.

13. Shen, Y.F. Handbook of Chinese liquor making technology (1st ed.) Beijing: 2007, China Light Industry Press.

14. Cobo-Díaz, J.F., Baroncelli, R., Le, F.G., \& Picot, A. Combined Metabarcoding and Co-occurrence Network Analysis to Profile the Bacterial, Fungal and Fusarium Communities and Their Interactions 
in Maize Stalks. Frontiers in Microbiology.2019, 10, 261, doi: 10.3389/fmicb.2019.00261.

15. Stéphanie, G., Francisco, D.A., Tieleman, B.I., \& Joana F.S. Dynamics of bacterial and fungal communities associated with eggshells during incubation. Ecology and evolution.2014, 4(7), 114057, doi: 10.1002/ece3.1011.

16. Yan, S.B., Tong, Q.Q. \& Guang, J.Q. Yeast dynamics and changes in volatile compounds during the fermentation of the traditional Chinese strong-flavor Daqu. LWT-Food Sci Technol.2019, 106, 57-63, doi: 10.1016/j.Iwt.2019.02.058.

\section{Figures}




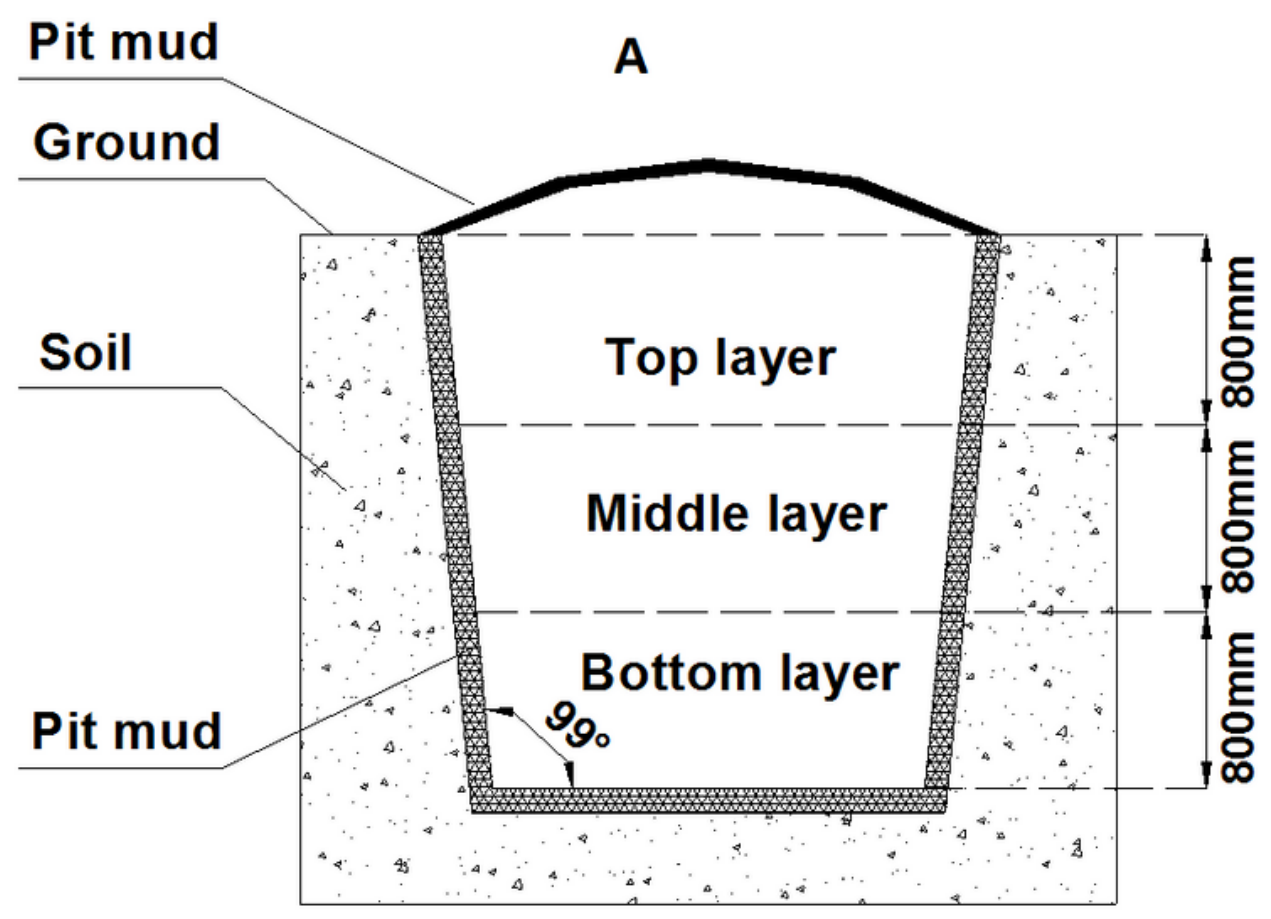

B

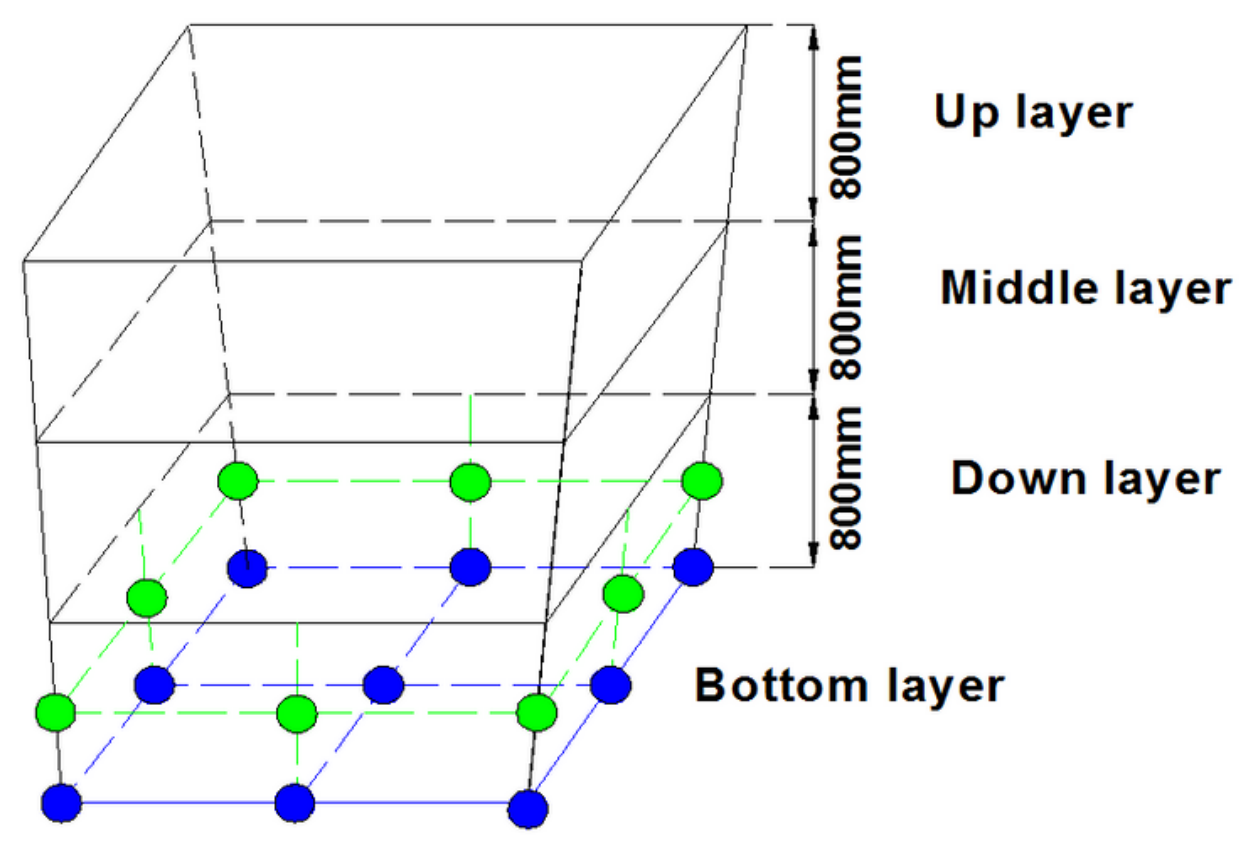

Figure 1

The profiles of the Chinese strong-flavour liquor pit mud (A) and the sampling sites of pit mud (B). 


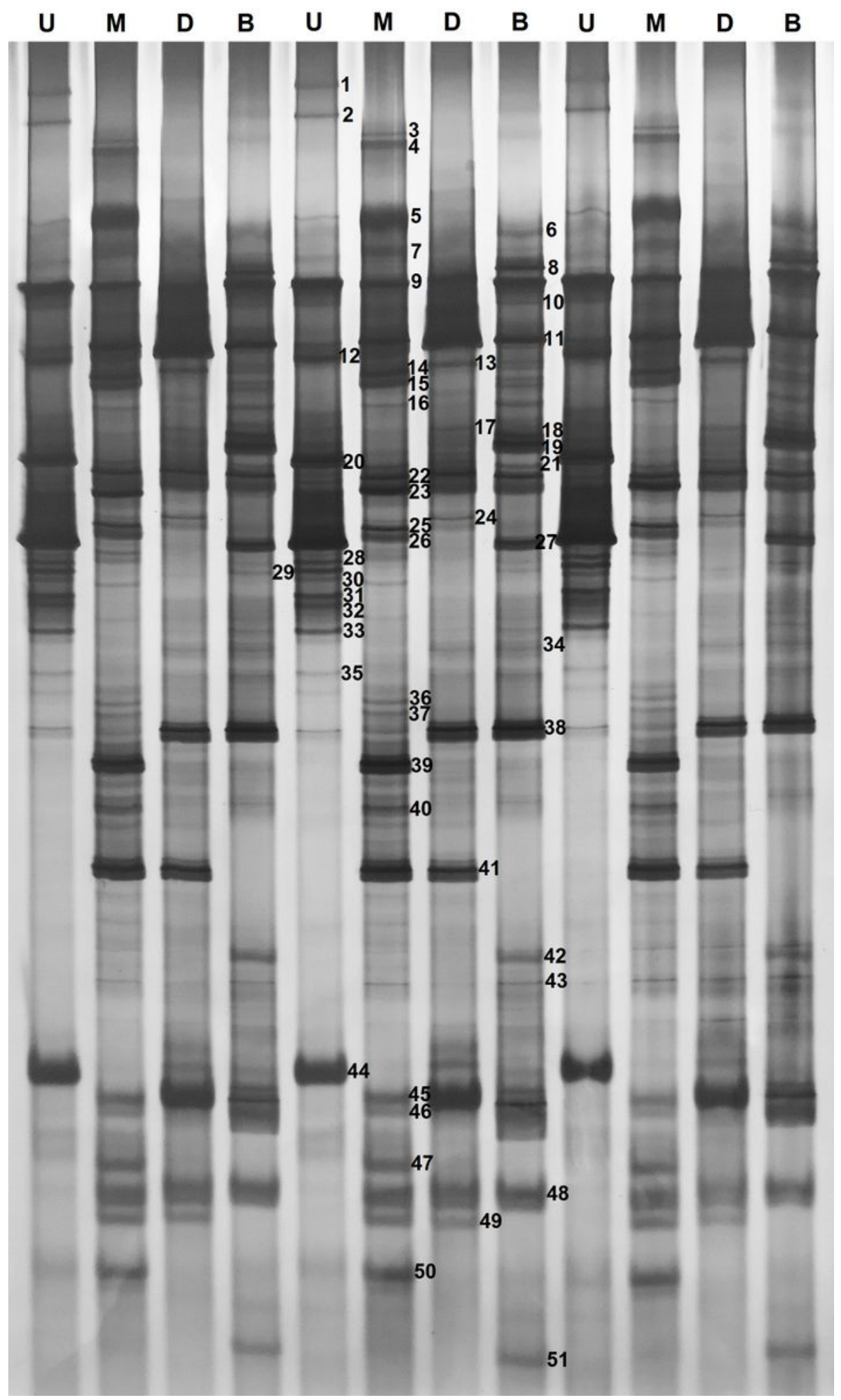

Figure 2

PCR-DGGE fingerprints of ITS gene extracted from fungal community in the pit mud samples collected from different spatial positions of cellar. Lanes $U, M, D$, and $B$ represent samples collected from up wall layer of cellar, middle wall layer of cellar, down wall layer of cellar, and bottom layer of cellar, respectively. The bands indicated with numbers were excised and sequenced and the alignment results are listed in Table 2. 


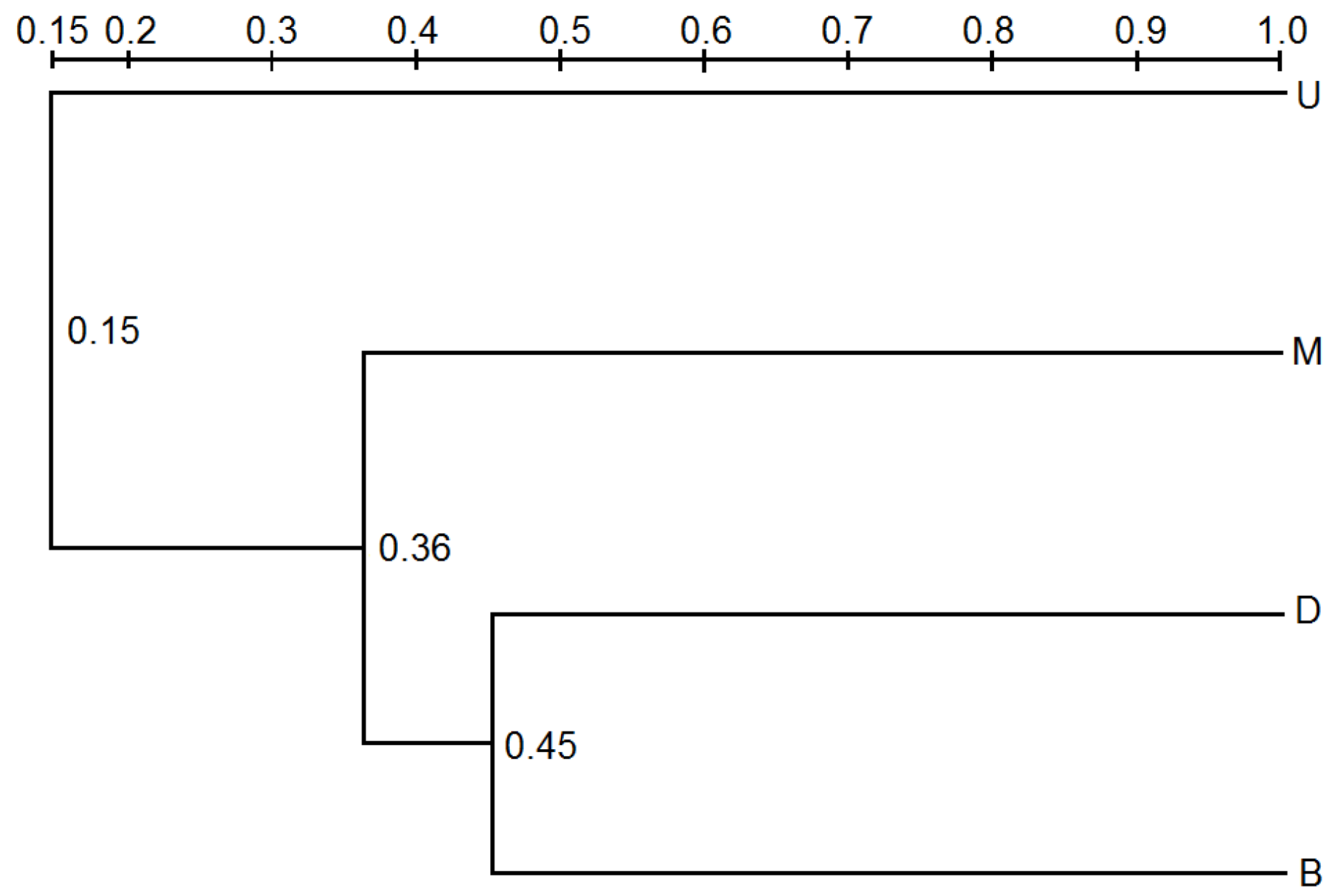

\section{Figure 3}

Clustering analysis of fungal DGGE profiles. Similarity was calculated using Euclidean distance and clustering was done using UPGMA. U, M, D, and B represent samples from up wall layer of cellar, middle wall layer of cellar, down wall layer of cellar, and bottom layer of cellar. 


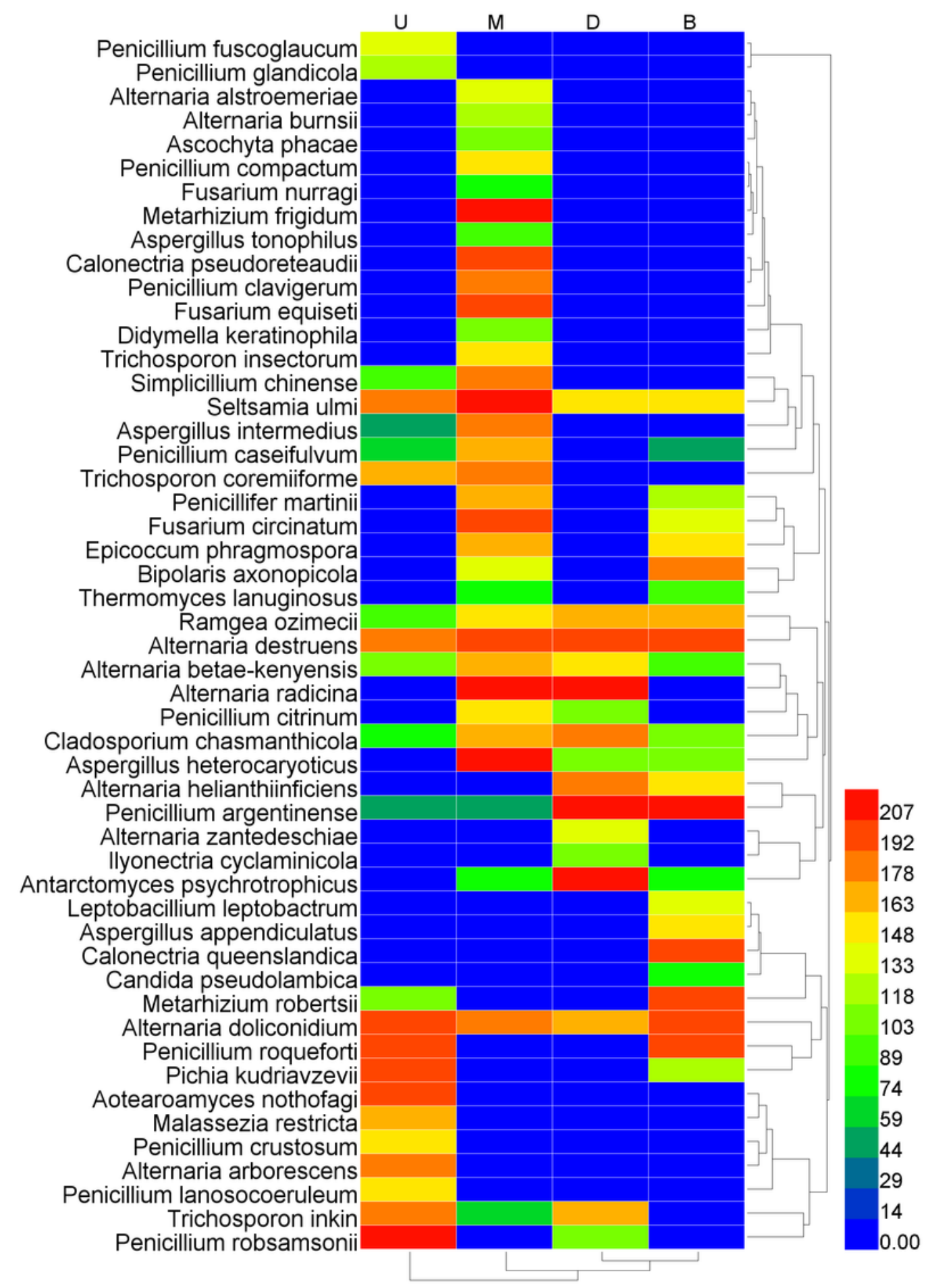

\section{Figure 4}

Heatmap of fungal communities in the pit mud samples from different spatial positions of cellar. Lanes $\mathrm{U}, \mathrm{M}, \mathrm{D}$, and $\mathrm{B}$ respectively represent pit mud samples collected from up wall layer of cellar, middle wall layer of cellar, down wall layer of cellar, and bottom layer of cellar, and were sampled from the same fermentation cellar. 


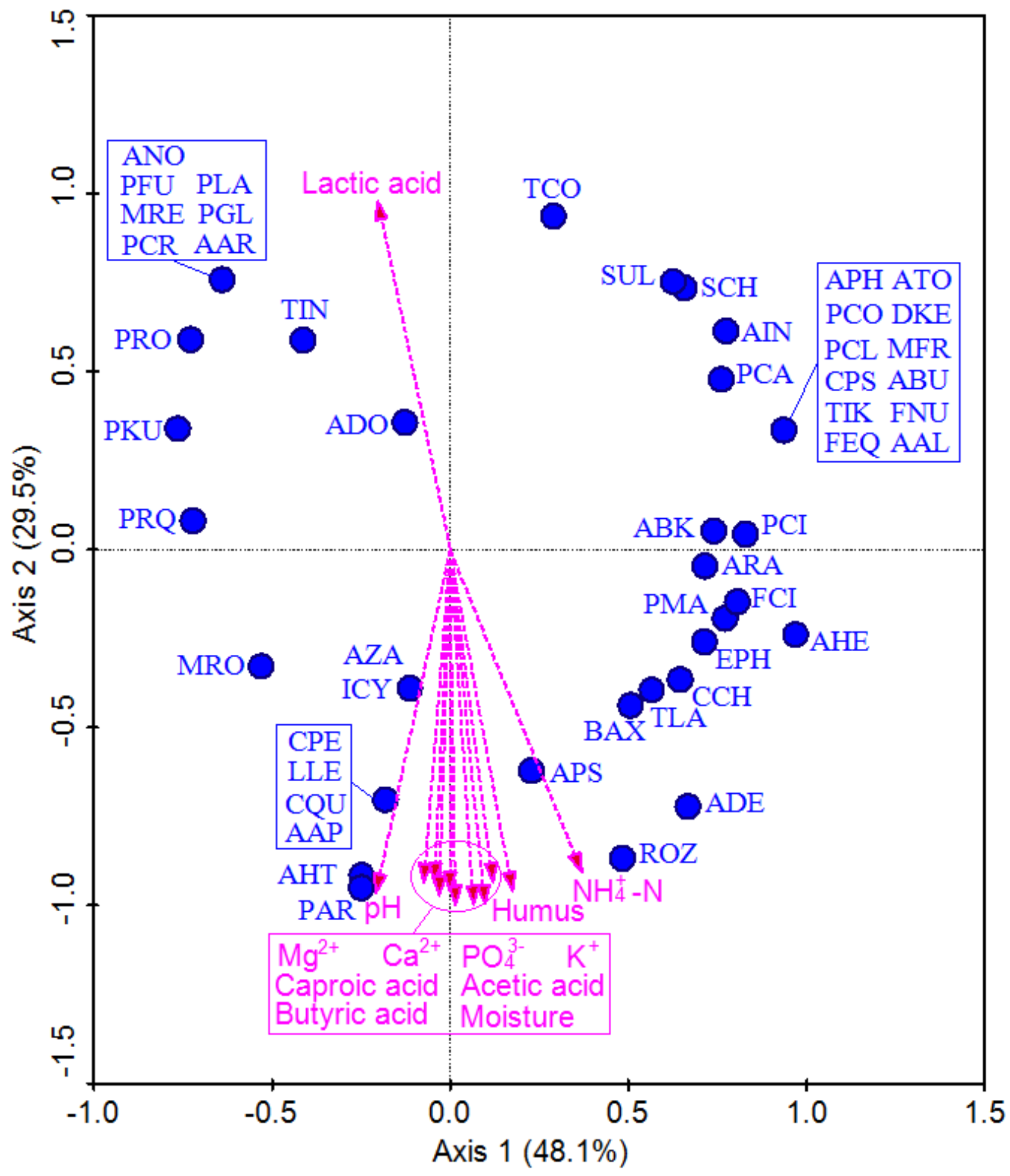

Figure 5

Redundancy analysis of fungal communities and physico-chemical attributes. The arrows indicate the direction and magnitude of biogeochemical attributes associated with fungal community structures. AAL: Alternaria alstroemeriae, AAP: Aspergillus appendiculatus, AAR: Alternaria arborescens, ABK: Alternaria betae-kenyensis, $A B U$ : Alternaria burnsii, ADE: Alternaria destruens, ADO: Alternaria dol-iconidium, AHE: Alternaria helianthiinficiens, AHT: Aspergillus heterocaryoticus, APH: Ascochyta phacae, APS: 
Antarctomyces psychrotrophicus, AIN: Aspergillus intermedius, ANO: Aotearoamyces nothofagi, ARA: Alternaria radicina, ATO: Aspergillus tonophilus, AZA: Alternaria zantedeschiae, BAX: Bipolaris axonopicola, $\mathrm{CCH}$ : Cladosporium chasmanthicola, CPS: Calonectria pseudoreteaudii, CPE: Candida pseudolambica, CQU: Calonectria queenslandica, DKE: Didymella keratinophila, EPH: Epicoccum phragmospora, FEQ: Fusarium equiseti, FCl: Fusarium circinatum, FNU: Fusarium nurragi, ICY: Ilyonectria cyclaminicola, LLE: Leptobacillium leptobactrum, MFR: Metarhizium frigidum, MRE: Malassezia restricta, MRO: Metarhizium roberts, PAR: Penicillium argentinense, PCA: Penicillium ca-seifulvum, PCI: Penicillium citrinum, PFU: Penicillium fuscoglaucum, PCL: Penicillium clavigerum, PCO: Penicillium compactum, PCR: Penicillium crustosum, PRO: Penicillium robsamsonii, PRQ: Peni-cillium roqueforti, PGL: Penicillium glandicola, PKU: Pichia kudriavzevii, PLA: Penicillium lanoso-coeruleum, PMA: Penicillifer martinii, ROZ: Ramgea ozimecii, SCH: Simplicillium chinense, SUL: Seltsamia ulmi, TCO: Trichosporon coremiiforme, TIN: Trichosporon insectorum, TIK: Trichosporon inkin, TLA: Thermomyces lanuginosus. 